\title{
Determinação de elementos- traço em testemunho de firn antártico usando ICP-MS
}

\section{Franciele Schwanck ${ }^{1 *}$ \\ Jefferson Cardia Simões ${ }^{1}$ \\ Michael Handley ${ }^{2}$ \\ Gino Casassa ${ }^{3}$}

\begin{abstract}
${ }^{1}$ Centro Polar e Climático do Instituto de Geociências da Universidade Federal do Rio Grande do Sul (UFRGS) - Porto Alegre (RS), Brasil.
\end{abstract}

${ }^{2}$ Climate Change Institute, University of Maine Orono (ME), Estados Unidos.

${ }^{3}$ Universidad de Magallanes - Punta Arenas, Chile.

*Autor correspondente: franschwanck@yahoo. com.br

\begin{abstract}
Resumo
Avaliar alterações nas concentrações de elementos-traço na neve da Antártica durante os últimos anos é de grande interesse para determinar em que medida as regiões mais remotas do nosso planeta estão sendo afetadas por emissões globais. Os elementos-traço estudados nesse trabalho foram determinados em um testemunho de firn da Antártica usando espectrometria de massas por plasma indutivamente acoplado. As concentrações obtidas para Al, As, Bi, Ca, Cd, Co, Cr, Fe, Li, Mg, Mn, Na, $\mathrm{Pb}, \mathrm{S}, \mathrm{Sr}$ e Ti são controladas pelas variações climáticas sazonais (verão/inverno), pela distância de transporte e pelas fontes naturais e antrópicas desses aerossóis. Contribuições naturais de solo, oriundas principalmente da região de Patriot Hills, são importantes fontes para alumínio, ferro, manganês e titânio. Os aerossóis marinhos, provenientes do gelo marinho e transportados pelas massas de ar, são fontes importantes de sódio, magnésio, estrôncio e enxofre. Lítio e cálcio apresentaram aportes consideráveis tanto de poeira continental como de aerossóis marinhos. Emissões vulcânicas foram consideradas fontes significativas de arsênio, bismuto, cádmio, cobalto, cromo e chumbo, com sua contribuição variando desde $10 \%$ (chumbo) até $85 \%$ (bismuto). Os fluxos de deposição natural de chumbo, cromo, bismuto e arsênio representaram apenas uma pequena fração do total depositado na neve. Para esses elementos, as atividades antrópicas ligadas à mineração e à queima de combustíveis fósseis no Hemisfério Sul possivelmente constituem o principal fator responsável por sua mobilização e transporte. Palavras-chave: elementos-traço, testemunho de firn, Antártica, ICP-MS.
\end{abstract}

\section{Abstract}

To estimate changes in the concentrations of trace elements in Antarctic snow, precipitated in recent years, is of great interest to determine to what extent the most remote regions of our planet are being affected by global emissions. The trace elements studied in this work were measured in an Antarctic firn core using inductively coupled plasma mass spectrometry. The concentrations obtained for $\mathrm{Al}, \mathrm{As}, \mathrm{Bi}, \mathrm{Ca}, \mathrm{Cd}$, Co, Cr, $\mathrm{Fe}, \mathrm{Li}$, $\mathrm{Mg}, \mathrm{Mn}, \mathrm{Na}, \mathrm{Pb}, \mathrm{S}, \mathrm{Sr}$ and Ti are controlled by seasonal climatic variations (summer) winter), by transport distance and by natural and anthropogenic sources of aerosols. Natural contributions of the soil, originating primarily from the Patriot Hills region, are important sources for aluminum, iron, manganese and titanium. Marine aerosols, formed from the surface of the sea ice cover and transported by air masses, are important sources of sodium, magnesium, strontium and sulfur. The elements lithium and calcium showed considerable contributions from continental dusts as well as marine aerosols. Volcanic emissions were considered important sources of arsenic, bismuth, cadmium, cobalt, chromium and lead, with a contribution ranging from $10 \%$ (lead) to $85 \%$ (bismuth). The flows of natural deposition of lead, chromium, bismuth and arsenic represented only a small fraction of the total amount deposited in the snow. For these elements, human activities related to mining and burning of fossil fuels in the Southern Hemisphere are possibly the main factor responsible for their mobilization and transport.

Keywords: trace elements, firn core, Antarctica, ICP-MS. 


\section{INTRODUÇÃO}

Elementos-traço são comuns em todos os ambientes e constantemente reciclados por intemperismo químico e mecânico, bem como por remobilização por meio de atividades humanas, o que acelera a sua velocidade de emissão (Barbante et al. 2011). Alguns desses elementos são considerados essenciais para a vida (e.g., Fe), outros são micronutrientes (e.g., Cr), enquanto outros são tóxicos em qualquer quantidade (e.g., $\mathrm{Pb}$ ). O impacto das atividades humanas nas emissões atmosféricas começa a ser perceptível com o início da fundição dos metais (Nriagu 1996). Embora existam indícios desse impacto a partir da Idade do Bronze, ele se tornou mais evidente durante os Impérios Grego e Romano, onde aparecem sinais claros do aumento das concentrações atmosféricas de chumbo, cobre e outros elementos-traço no gelo da Groenlândia (Hong et al. 1994), em sedimentos lacustres na Europa (Renberg et al. 2002) e em turfeiras (Shotyk et al. 1998, Martínez-Cortizas et al. 1999).

A fim de tentar compreender as interferências humanas na ciclagem de elementos-traço (especialmente chumbo, arsênio, cobre, cádmio etc.) na atmosfera e seu impacto sobre as regiões polares, análises em testemunhos de gelo são amplamente utilizadas. Trabalhos realizados em Terra de Coats no setor atlântico da Antártica (Wolff \& Suttie 1994, Wolff et al. 1999, Planchon et al. 2002a, 2002b), na Terra Victoria e no domo Law no setor do Oceano Índico (Vallelonga et al.2002, Van de Velde et al. 2005, Burn-Nunes et al. 2011) forneceram evidências de

\section{MATERIAIS E MÉTODOS}

\subsection{Sítio de amostragem}

Em dezembro de 2004, uma equipe formada por pesquisadores chilenos e brasileiros extraiu seis testemunhos de firn e gelo do continente antártico ao longo de uma travessia entre o Polo Sul Geográfico $\left(90^{\circ} \mathrm{S}\right)$ e a estação chilena Tenente Parodi $\left(80^{\circ} 18^{\prime} \mathrm{S}, 81^{\circ} 22^{\prime} \mathrm{W}\right)$. A extração dos testemunhos foi realizada a cada 220 quilômetros. A Figura 1 mostra a localização dos testemunhos perfurados. Este trabalho discute os resultados das análises realizadas no testemunho IC-6.

O testemunho IC-6 foi perfurado na coordenada $81^{\circ} 03^{\prime} 10^{\prime \prime} \mathrm{S}$ e $79^{\circ} 50^{\prime} 09^{\prime}$ ' W, a $750 \mathrm{~m}$ de altitude. A taxa de acumulação anual média para o local de coleta foi de $0,30 \mathrm{~m}$ eq. $\mathrm{H}_{2} \mathrm{O}^{-1}$. Esse testemunho apresenta profundidade variações nas concentrações de vários elementos-traço. Tais estudos identificaram que o aumento das emissões a partir do Hemisfério Sul teve início por volta de 1880 para $\mathrm{Pb}$ e no começo do século XX para outros metais, tais como $\mathrm{Cr}, \mathrm{Cu}, \mathrm{Ag}, \mathrm{Bi}$ e $\mathrm{U}$, com um rápido aumento nas últimas décadas.

No presente trabalho, serão apresentadas as concentrações de 16 elementos-traço de um testemunho de firn $^{1}$ antártico coletado durante o verão de 2004/2005. O testemunho foi subamostrado utilizando-se cuidadoso protocolo de descontaminação em câmara fria $\left(-20^{\circ} \mathrm{C}\right)$ e derretido em um sistema de fusão contínua (localizado em sala limpa classe 100). As subamostras foram analisadas por espectrometria de massas com plasma indutivamente acoplado (Inductively Coupled Plasma Mass Spectrometry, ICP-MS). O registro dos elementos-traço em testemunhos de neve, firn e gelo tem se mostrado uma das melhores ferramentas para estudos paleoambientais com objetivo de reconstruir o impacto das emissões para a atmosfera ao longo do tempo (Legrand \& Mayewski 1997). O conteúdo de elementos-traço tem sido frequentemente usado para determinar fontes de aerossóis (Hong et al. 1998, Gabrielli et al.2005b), entender ciclos biogeoquímicos (Delmas 1995) e avaliar o impacto de emissões antrópicas (Planchon et al. 2002b, Barbante et al. 2004) e vulcânicas (Matsumoto \& Hinkley 2001, Vallelonga et al. 2003) sobre o passado e o presente da atmosfera. de 35,06 m e foi extraído do setor ocidental do manto de gelo antártico. Ele está a uma distância de $85 \mathrm{~km}$ das montanhas Ellsworth em Patriot Hills.

A presença das montanhas exerce uma forte influência sobre o padrão de acumulação, provavelmente por divergência no fluxo de neve movimentado pelo vento, o que resulta em acúmulo reduzido. A taxa de acúmulo aumenta à medida que cresce a distância das montanhas, com ablação ocorrendo sobre o campo de gelo azul e acumulação na área de geleiras (Casassa et al. 1998). Isso demonstra que o gelo flui para nordeste de Patriot Hills, rumo à plataforma de gelo Ronne. Este também é o destino do fluxo do gelo do local onde foi feita a perfuração do testemunho IC-6.

\footnotetext{
'Estágio intermediário entre a neve e o gelo. O limite entre a neve e o firn não é bem definido, geralmente é identificado por características tais como cristais soldados uns aos outros, mas onde ainda persiste a conexão dos espaços intergranulares (ou seja, ainda é permeável). O limite firn-gelo, por sua vez, é marcado pelo fechamento da conexão entres os poros e ocorre quando a densidade atinge $0,83 \mathrm{~g} / \mathrm{cm}^{-3}$ (Simões 2004 ).
} 


\section{Figura 1}

Mapa da Antártica com destaque para os pontos de coleta de testemunhos de gelo durante a expedição chileno-brasileira (círculos, IC-1 a IC-6) e as principais estações científicas (estrelas). Outros sítios de coletas de testemunhos de gelo citados neste trabalho também são indicados (quadrados) (figura adaptada de Simões, 2011).

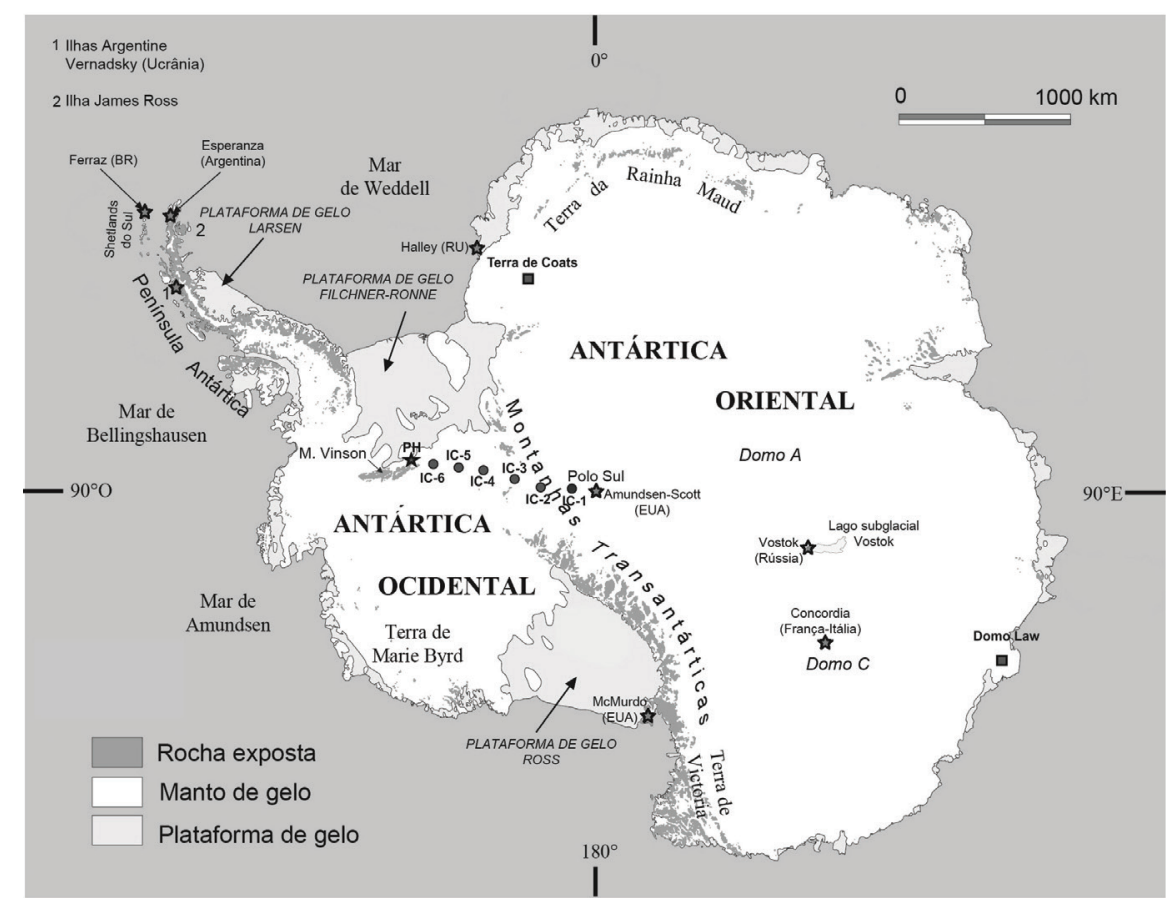

\subsection{Procedimentos analíticos}

Os trabalhos de preparação de amostras e descontaminação foram realizados no Climate Change Institute (CCI) da Universidade do Maine, em Orono, nos Estados Unidos. A descontaminação do testemunho de firn IC-6 foi realizada em câmara fria a $-20^{\circ} \mathrm{C}$ pela raspagem manual (entre 2 e $4 \mathrm{~mm}$ ) da parte externa do testemunho com faca de cerâmica (procedimento realizado conforme Tao et al.2001). As extremidades de cada seção foram removidas por serem os locais mais suscetiveis a contaminações. Após a descontaminação, as seções foram enviadas para uma sala limpa classe 100, onde foram subamostradas usando o sistema de fusão contínua desenvolvido pela equipe do CCI (mais detalhes em Osterberg et al. 2006). Imediatamente após a fusão, todas as amostras foram acidificadas com $\mathrm{HNO}^{3}$ bidestilado a $1 \%$.

As concentrações dos elementos-traço $\left({ }^{27} \mathrm{Al},{ }^{75} \mathrm{As},{ }^{209} \mathrm{Bi}\right.$, ${ }^{44} \mathrm{Ca},{ }^{111} \mathrm{Cd},{ }^{59} \mathrm{Co},{ }^{52} \mathrm{Cr},{ }^{56} \mathrm{Fe},{ }^{7} \mathrm{Li},{ }^{24} \mathrm{Mg},{ }^{55} \mathrm{Mn},{ }^{23} \mathrm{Na},{ }^{208} \mathrm{~Pb}$, ${ }^{32} \mathrm{~S},{ }^{88} \mathrm{Sr} \mathrm{e}^{47} \mathrm{Ti}$ ) foram determinadas pelo ICP-MS Thermo Electron Element 2 do CCI em 1.378 amostras de acordo com as configurações descritas na Tabela 1 . O uso de um sistema de introdução de amostra Apex ESI aumentou a sensibilidade do aparelho e reduziu a formação de óxidos no plasma, reduzindo os limites de detecção e permitindo que isótopos menos abundantes fossem medidos. $\mathrm{O}$ amostrador automático CETAC (Omaha, NE) Modelo ASX 260 está localizado dentro de uma capela HEPA classe 100 ao lado do ICP-MS para reduzir ainda mais o risco de contaminação.

O ICP-MS foi calibrado diariamente com cinco padrões adequados ao intervalo de concentração da amostra esperado. Embora não exista nenhum material de referência certificado para elementos-traço em gelo polar, as análises com padrão de água fluvial (SLRS-4, Conselho Nacional de Pesquisa do Canadá, Ottawa, Canadá) produzem concentrações dentro da faixa de certificação, confirmando a exatidão do método (detalhes em Osterberg et al. 2006).

\begin{tabular}{|c|c|c|}
\hline & Instrumento & Thermo Electron Element 2 \\
\hline & Potência & $1.280 \mathrm{~W}$ \\
\hline & Vazão de gás do plasma & $16 \mathrm{~L} / \mathrm{min}$ \\
\hline & Vazão auxiliar de argônio & $0,85 \mathrm{~L} / \mathrm{min}$ \\
\hline & Vazão de gás do nebulizador & $0,8 \mathrm{~L} / \mathrm{min}$ \\
\hline & Cone amostrador & $\mathrm{Ni} ; 1,1 \mathrm{~mm}$ \\
\hline & Cone skimmer & $\mathrm{Ni} ; 0,8 \mathrm{~mm}$ \\
\hline & Sistema de introdução de amostra & ESI ApexQ \\
\hline Tabela 1 & Nebulizador & ESI PFA-ST \\
\hline ICP-MS & Resolução (m/Dm) & Baixa: 400; média: 4.000 e alta: 10.000 \\
\hline quisição & Taxa de absorção da amostra & $100 \mu \mathrm{L} / \min$ \\
\hline lemen- & Sensibilidade & $800.000 \mathrm{cps}$ (contagem por segundo) para $100 \mathrm{ng} / \mathrm{L}{ }^{115} \mathrm{ln}$ \\
\hline de gelo. & Tempo total de medição & $2 \min 20 \mathrm{~s}$ \\
\hline
\end{tabular}




\subsection{Datação das amostras}

Para a datação do testemunho IC-6, utilizou-se a contagem de camadas anuais baseada nas variações sazonais das concentrações elementares, ilustradas na Figura 2. A partir das medidas de concentrações elementares determinadas por ICP-MS e do conteúdo iônico por cromatografia (Hammes 2011), foram identificados os picos anuais sazonais, correspondentes às estações secas de inverno austral, e aferidas as datas de deposição sequencialmente. Durante o inverno austral, a extensão do gelo marinho ao redor do continente atinge seu máximo e dificulta a penetração das massas de ar no platô antártico, o que ocasiona temperaturas ainda mais baixas e precipitação seca (King \& Turner 1997). Em amostras polares, a datação por esse tipo de contagem é confiável, uma vez que nessas regiões o conteúdo depositado durante o verão e inverno é preservado, pois processos como derretimento, percolação e recongelamento são raros. No caso de picos de concentração duvidosos, a comparação com os perfis de cromatografia iônica (Hammes 2011) permitiu resolver a posição correta. Além disso, o espaçamento entre picos vizinhos apresenta certa regularidade que também permite inferir a posição correta. A datação do testemunho IC-6 inicia-se no ano de 2002 e a contagem dos picos referentes à estação seca (inverno) originou 68 anos de registro, chegando ao ano de 1934, ou seja, uma taxa anual de acumulação de neve média de $0,30 \mathrm{~m}$ de água.

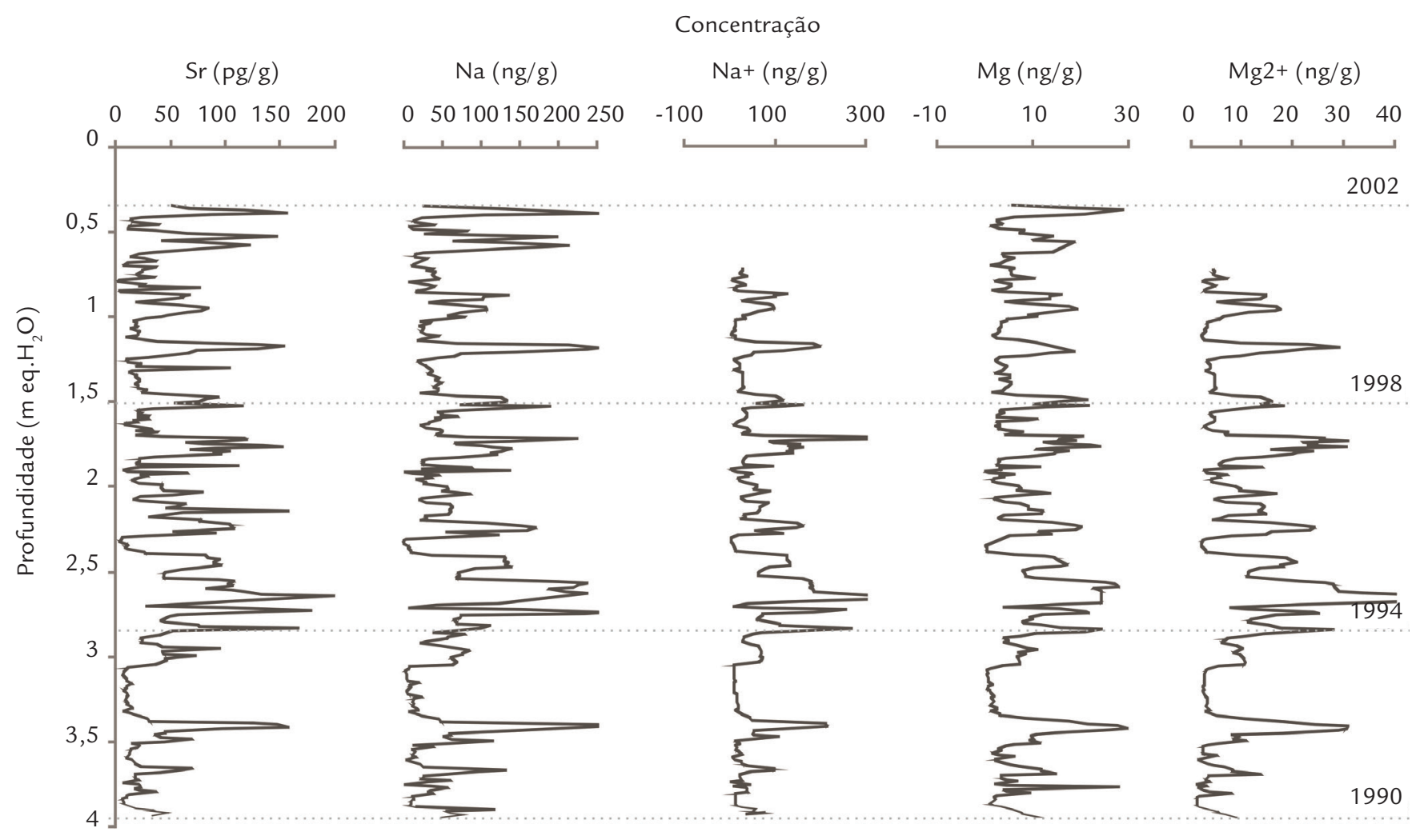

\section{RESULTADOS E DISCUSSÕES}

\subsection{Concentrações elementares}

Foram medidas as concentrações de 16 elementos (Al, As, Bi, Ca, Cd, Co, Cr, Fe, Li, Mg, Mn, Na, Pb, S, Sr e Ti) em 1.378 amostras. Essas amostras correspondem ao intervalo
Figura 2

Datação relativa com base nas concentrações elementares de $\mathrm{Na}, \mathrm{Mg}$ e Sr e nas concentrações iônicas de $\mathrm{Na}^{+}$e $\mathrm{Mg}^{2+}$, segundo Hammes (2011). Pode-se observar uma boa correlação entre os elementos $\mathrm{Na}$ e $\mathrm{Mg}$ medidos por métodos diferentes. de profundidade entre a superfície e 35,06 metros, perfazendo cerca de 68 anos de registro contínuo. Para correção de eventuais contaminações durante o processo de fusão, foram 
usadas amostras de água deionizada (brancos). Os brancos foram analisados juntamente com as amostras, e as concentrações elementares determinadas nos brancos foram subtraídas das amostras (Knüsel et al. 2003, Rhodes 2011). O resumo das concentrações é apresentado na Tabela 2.

As concentrações medidas variam em ordem de magnitude de um elemento para outro. As maiores concentrações são na ordem do ng/g, observadas para $\mathrm{Al}, \mathrm{Ca}, \mathrm{Fe}, \mathrm{Mg}$, $\mathrm{Na}$ e $\mathrm{S}$, principais constituintes de poeira continental e do aerossol marinho. Concentrações na ordem do pg/g são observadas para os demais elementos.

Os perfis de concentração (e.g., Mn, Figura 3) apresentam uma forte sazonalidade, que é atribuída aos regimes anuais de precipitação na região, com altas concentrações relativas durante a estação seca (inverno austral) e baixas concentrações durante a estação úmida (verão austral).
As concentrações mais elevadas observadas na estação seca são consequência de dois fatores: menor precipitação, o que facilita a mobilização do aerossol, uma vez que as forças de coesão entre partículas de solo são mínimas, e a diminuição da disponibilidade de radiação solar, reduzindo efeitos de sublimação. Essa sazonalidade é observada na maior parte das concentrações elementares medidas.

Observando a Tabela 3, nota-se que as concentrações elementares medidas no testemunho de firn IC-6 têm, de maneira geral, valores compatíveis com as medidas em outros locais da Antártica. As pequenas variações observadas podem estar relacionadas a diferenças entre os locais de amostragem (altitude, circulação atmosférica, precipitação e taxa de acumulação), bem como à proximidade de fontes naturais de ressuspensão de poeira do solo, mas não se pode excluir as diferenças na preparação de amostras e os métodos de análise.

Tabela 2

Resumo estatístico das concentrações dos 16 elementos determinados no testemunho de firn IC-6.

\begin{tabular}{lccccc}
\hline Elementos & $\begin{array}{c}\text { Concentração } \\
\text { média (mín-máx) }\end{array}$ & Mediana & $\begin{array}{c}\text { Desvio } \\
\text { padrão }( \pm)\end{array}$ & Erro $( \pm)$ & $\begin{array}{c}\text { Número de } \\
\text { amostras }\end{array}$ \\
\hline $\mathrm{Al}(\mathrm{ng} / \mathrm{g})$ & $1,10(<0,01-18,08)$ & 0,52 & 1,91 & 0,05 & 1.352 \\
\hline $\mathrm{As}(\mathrm{pg} / \mathrm{g})$ & $1,88(<0,01-9,49)$ & 1,45 & 1,40 & 0,04 & 1.350 \\
\hline $\mathrm{Bi}(\mathrm{pg} / \mathrm{g})$ & $0,34(<0,01-9,79)$ & 0,10 & 0,96 & 0,03 & 1.349 \\
\hline $\mathrm{Ca}(\mathrm{ng} / \mathrm{g})$ & $4,85(<0,01-29,16)$ & 3,40 & 4,54 & 0,12 & 1.342 \\
\hline $\mathrm{Cd}(\mathrm{pg} / \mathrm{g})$ & $0,28(<0,01-1,89)$ & 0,18 & 0,32 & 0,01 & 1.341 \\
\hline $\mathrm{Co}(\mathrm{pg} / \mathrm{g})$ & $1,00(<0,01-6,84)$ & 0,66 & 1,06 & 0,03 & 1.352 \\
\hline $\mathrm{Cr}(\mathrm{pg} / \mathrm{g})$ & $3,92(<0,01-39,92)$ & 1,59 & 5,91 & 0,16 & 1.335 \\
\hline $\mathrm{Fe}(\mathrm{ng} / \mathrm{g})$ & $0,39(<0,01-5,95)$ & 0,16 & 0,68 & 0,02 & 1.356 \\
\hline $\mathrm{Li}(\mathrm{pg} / \mathrm{g})$ & $2,56(<0,01-12,84)$ & 1,96 & 2,28 & 0,06 & 1.331 \\
\hline $\mathrm{Mg}(\mathrm{ng} / \mathrm{g})$ & $9,87(<0,01-29,77)$ & 8,19 & 6,93 & 0,19 & 1.305 \\
\hline $\mathrm{Mn}(\mathrm{pg} / \mathrm{g})$ & $15,44(<0,01-69,17)$ & 12,15 & 12,32 & 0,34 & 1.336 \\
\hline $\mathrm{Na}(\mathrm{ng} / \mathrm{g})$ & $65,43(<0,01-275,82)$ & 45,89 & 58,89 & 1,62 & 1.327 \\
\hline $\mathrm{Pb}(\mathrm{pg} / \mathrm{g})$ & $5,03(<0,01-36,40)$ & 3,63 & 5,21 & 0,14 & 1.338 \\
\hline $\mathrm{S}(\mathrm{ng} / \mathrm{g})$ & $14,10(1,25-42,29)$ & 11,85 & 8,63 & 0,24 & 1.298 \\
\hline $\mathrm{Sr}(\mathrm{pg} / \mathrm{g})$ & $55,87(1,08-199,69)$ & 42,88 & 41,96 & 1,16 & 1.314 \\
\hline $\mathrm{Ti}(\mathrm{pg} / \mathrm{g})$ & $28,61(<0,01-343,61)$ & 16,61 & 36,14 & 0,99 & 1.343 \\
\hline & & & & &
\end{tabular}

Profundidade ( $m$ eq. $\mathrm{H}_{2} \mathrm{O}$ )

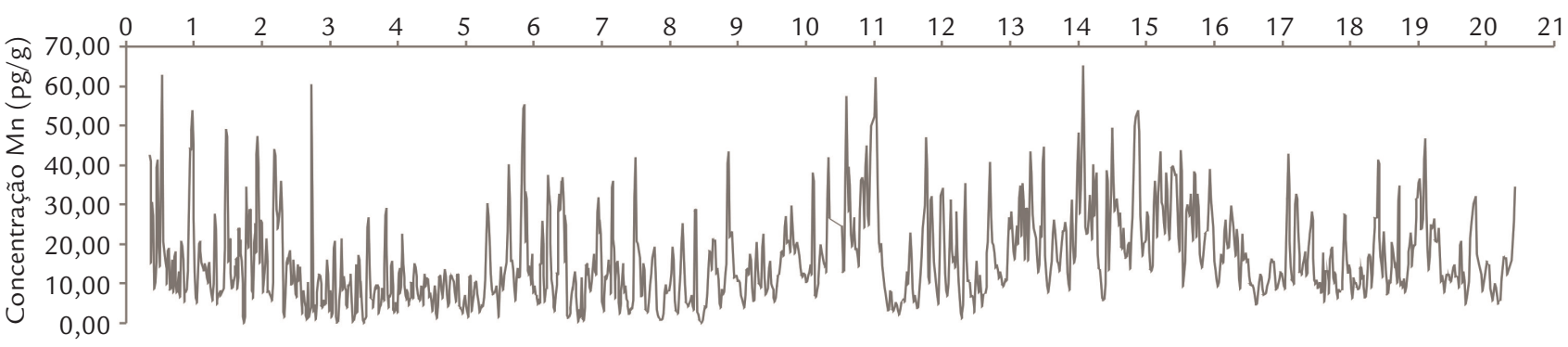

Figura 3

Perfil de concentração do elemento manganês (em pg/g). 


\begin{tabular}{|c|c|c|c|c|c|c|c|c|c|c|c|c|c|c|c|c|}
\hline \multicolumn{17}{|c|}{ Concentração média (pg/g) } \\
\hline & $\mathrm{Al}^{*}$ & As & $\mathrm{Bi}$ & $\mathrm{Ca}^{*}$ & $\mathrm{Cd}$ & Co & $\mathrm{Cr}$ & $\mathrm{Fe}^{*}$ & $\mathrm{Li}$ & Mg* & Mn & $\mathrm{Na}^{*}$ & $\mathrm{~Pb}$ & $\mathrm{~S}^{*}$ & Sr & $\mathrm{Ti}$ \\
\hline IC-6 (1934-2002) & 1,10 & 1,88 & 0,34 & 4,85 & 0,28 & 1,00 & 3,92 & 0,39 & 2,56 & 9,87 & 15,44 & 65,43 & 5,03 & 14,10 & 55,87 & 28,61 \\
\hline $02-6(1955-1975)^{* *}$ & 2,07 & 3,48 & 2,71 & 2,29 & 4,07 & 22,55 & 11,87 & 0,90 & 9,86 & 6,48 & 25,33 & 6,60 & 12,07 & 6,60 & 8,88 & 22,33 \\
\hline Domo Law $(1932-1989)^{* * * *}$ & - & - & 0,05 & 1,85 & 0,24 & 0,92 & - & - & - & - & 5,26 & 50,69 & 1,86 & - & 66,21 & - \\
\hline Terra de Coats $(1958-1975)^{* * * * *}$ & 0,52 & - & 0,06 & - & 0,20 & 0,41 & 1,91 & - & - & - & 10,37 & - & 3,73 & - & - & - \\
\hline
\end{tabular}

*Concentrações em ng/g.

Fontes: ${ }^{*}$ Dixon et al. 2013; ***Do Hur et al. 2007; ****Vallelonga et al. 2004; $* * * *$ Planchon et al. 2002b.

A fim de se diferenciar a importância das contribuições naturais e antropogênicas para as amostras do testemunho IC-6, tentou-se separar as possíveis contribuições de diferentes fontes naturais, incluindo poeira continental, spray marinho e emissões vulcânicas.

A contribuição de poeira de rocha e solo foi determinada a partir do fator de enriquecimento crustal $\left(\mathrm{FE}_{\mathrm{c}}\right)$, que foi calculado de acordo com a seguinte equação apresentada em Osterberg (2007):

$\mathrm{FE}_{\mathrm{C}}=\frac{\mathrm{X}_{\text {gelo }} / \mathrm{Mn}_{\text {gelo }}}{\mathrm{X}_{\text {ref }} / \mathrm{Mn}_{\text {ref }}}$

onde $\mathrm{X}_{\text {gelo }}$ é a concentração na amostra do elemento para o qual se busca o fator de enriquecimento, $\mathrm{Mn}_{\text {gelo }}$ é a concentração de manganês na amostra, $\mathrm{X}_{\text {ref }}$ é a concentração do elemento no material de referência a ser utilizado e $\mathrm{Mn}_{\text {ref }}$ é a concentração de manganês no material de referência.

Após a análise de várias opções, decidiu-se utilizar o manganês como elemento de referência, uma vez que esse é considerado um bom indicador indireto (proxy) de poeira continental (Gabrielli et al. 2005a, Marteel 2007). Neste trabalho, as razões de concentrações elementares de referência correspondem à composição média da crosta continental superior, valores obtidos a partir da literatura (Wedepohl 1995).

Para calcular a contribuição do spray marinho foi utilizado o fator de enriquecimento oceânico $\left(\mathrm{FE}_{\mathrm{o}}\right)$, calculado a partir da seguinte equação, segundo Dixon et al. (2013):

$F E_{o}=\frac{X_{\text {gelo }} / \mathrm{Na}_{\text {gelo }}}{X_{\text {ref }} / \mathrm{Na}_{\text {ref }}}$

onde $\mathrm{X}_{\text {gelo }}$ é a concentração na amostra do elemento para o qual se busca o fator de enriquecimento, $\mathrm{Na}_{\text {gelo }}$ é a concentração de sódio na amostra, $X_{\text {ref }}$ é a concentração do elemento no material de referência a ser utilizado e $\mathrm{Na}_{\text {ref }}$ é a concentração de sódio no material de referência.

O sódio foi escolhido como elemento de referência por ser o principal constituinte do sal marinho (Weller et al. 2008, Dixon et al. 2013). Neste trabalho, as razões de concentrações elementares de referência correspondem à composição média da água oceânica (Lide 2005).
Tabela 3

Concentração média de elementos-traço do testemunho IC-6 em comparação com outros trabalhos.

Para separar essas duas fontes potenciais, foram calculadas a fração marinha e crustal de cada elemento de acordo com a seguinte fórmula (Dixon et al.2013):

$X_{0}=N a_{\text {gelo }} \times\left(X_{\text {ref }} / \mathrm{Na}_{\text {ref }}\right)$

onde $\mathrm{X}_{\mathrm{O}}$ é a fração de origem oceânica do elemento, $\mathrm{Na}_{\text {gelo }}$ é a concentração de sódio na amostra, $\mathrm{X}_{\mathrm{ref}}$ é a concentraçã̃o do elemento no material de referência a ser utilizado e $\mathrm{Na}_{\text {ref }}$ é a concentração de sódio no material de referência.

A fração oceânica foi subtraída de cada amostra e então foi calculada a fração crustal utilizando a mesma fórmula, porém substituindo sódio por manganês como material de referência.

A contribuição vulcânica foi estimada a partir do enxofre e do sulfato não marinho (non sea-salt ou nss- $\mathrm{SO}_{4}{ }^{2-}$ ). Acredita-se que aproximadamente 10 a $15 \%$ desses elementos na atmosfera da Antártica são de origem vulcânica (Do Hur et al. 2007). Combinando esses números com estimativas das taxas de metal/S de emissões vulcânicas (Hinkley et al. 1999), foi possível calcular as entradas vulcânicas para alguns elementos-traço. Também foi usada a taxa de metal/S para a pluma do monte Erebus ( $77^{\circ} 32^{\prime}$ S, $\left.167^{\circ} 10^{\prime} \mathrm{E}\right)$, segundo Zreda-Gostynska et al. (1997), para representar as contribuições locais. Por falta de dados na literatura, não foi possível estimar a contribuição vulcânica da ilha Deception (62 $\left.{ }^{\circ} 57^{\prime} \mathrm{S}, 60^{\circ} 38^{\prime} \mathrm{W}\right)$, porém acredita-se que essas emissões são tão importantes quanto aquelas oriundas do monte Erebus. A média das contribuições de fontes naturais calculadas é apresentada na Tabela 4.

Dos elementos analisados, estima-se que Al, Fe, Mn e Ti são predominantemente originados de emissões a partir da crosta terrestre. Os elementos apresentam fator de enriquecimento crustal baixo, menor que a unidade de referência (nesse trabalho foi adotado 1 como valor de referência). A proximidade com as montanhas de Patriot Hills (a $85 \mathrm{~km}$ de distância) pode ser responsável por um aporte natural significativo de poeira continental e solo. $\mathrm{Mg}, \mathrm{Na}$ e Sr foram considerados de origem marinha, uma vez que o fator de enriquecimento oceânico apresentado é baixo (em torno de 1). Para Ca e Li, tanto os aerossóis continentais como os marinhos são importantes fontes contribuintes (fator de enriquecimento crustal e oceânico menor que 5). 


\begin{tabular}{|c|c|c|c|c|c|c|c|}
\hline \multirow{2}{*}{ Elementos } & \multirow{2}{*}{$\begin{array}{l}\text { Concentração } \\
\text { média }(p g / g)\end{array}$} & \multirow{2}{*}{$\begin{array}{l}\text { Contribuição } \\
\text { terrestre (\%) }\end{array}$} & \multirow{2}{*}{$\begin{array}{l}\text { Contribuição } \\
\text { marinha (\%) }\end{array}$} & \multicolumn{2}{|c|}{ Contribuição vulcânica global (\%) } & \multicolumn{2}{|c|}{ Contribuição vulcânica regional (\%) } \\
\hline & & & & Mínima & Máxima & Mínima & Máxima \\
\hline $\mathrm{Al}^{*}$ & 1,10 & 94,55 & 0,90 & & & 1,82 & 2,73 \\
\hline As & 1,88 & 3,19 & 1,06 & 2,13 & 3,19 & 20,21 & 33,51 \\
\hline $\mathrm{Bi}$ & 0,34 & 2,94 & 2,94 & 55,88 & 85,29 & & \\
\hline $\mathrm{Ca}^{*}$ & 4,85 & 25,35 & 74,04 & & & 0,20 & 0,41 \\
\hline $\mathrm{Cd}$ & 0,28 & 3,57 & 3,57 & 21,84 & 32,76 & 35,49 & 60,06 \\
\hline $\mathrm{Fe}^{*}$ & 0,39 & 92,32 & 2,56 & & & 2,56 & 2,56 \\
\hline $\mathrm{Li}$ & 2,56 & 36,78 & 63,22 & & & & \\
\hline $\mathrm{Mg}^{*}$ & 9,87 & 4,85 & 95,15 & & & & \\
\hline $\mathrm{Mn}$ & 15,44 & 75,33 & 0,06 & 14,57 & 21,89 & 1,62 & 2,72 \\
\hline $\mathrm{Na}^{*}$ & 65,43 & 1,18 & 98,70 & & & 0,03 & 0,05 \\
\hline $\mathrm{Ti}$ & 28,61 & 83,72 & 0,03 & & & 9,75 & 16,25 \\
\hline
\end{tabular}

Concentrações médias dos 16 elementos analisados no testemunho de firn IC-6 e contribuição marinha, crustal e vulcânica.

Em comparação, o fator de enriquecimento crustal para As, $\mathrm{Bi}, \mathrm{Cd}, \mathrm{Co}, \mathrm{Cr}, \mathrm{Pb}$ e $\mathrm{S}$ apresentou enriquecimento pelo menos uma ordem de magnitude maior do que aquele esperado para entradas de poeira e sal marinho, proporcionando uma forte indicação de que esses elementos apresentam outra fonte de contribuição. O fator de enriquecimento médio de Bi e Cd foi calculado em 106 e 105, respectivamente, e o vulcanismo pode ser uma importante fonte de emissão para os dois elementos, enquanto a média de enriquecimentos de $\mathrm{As}, \mathrm{Co}, \mathrm{Cr}$ e $\mathrm{Pb}$ está dentro da faixa 4-33, indicando que a crosta terrestre e/ou o oceano ainda são fontes de emissão importantes para esses elementos.

As estimativas da contribuição vulcânica mostram que as emissões vulcânicas globais são uma fonte importante de elementos-traço, sendo o bismuto o principal contribuinte (aproximadamente $85 \%$ ) nas amostras analisadas. Cádmio, cromo e cobalto também apresentam contribuições importantes, chegando a aproximadamente $33 \%, 31 \%$ e $48 \%$ respectivamente. A erupção do vulcão Cerro Hudson no Chile, em 1991, aparece registrada como pico nos perfis de concentração de bismuto e cromo e menos evidente no perfil de chumbo (Figura 4).

As emissões do monte Erebus são uma fonte importante de arsênio e cádmio, sendo responsáveis por 33\% e $60 \%$ da concentração desses elementos. Para os demais elementos-traço, a contribuição vulcânica varia entre $1 \mathrm{e}$ $15 \%$, indicando que os aerossóis vulcânicos não são fontes significativas desses elementos quando comparados com incrementos marinhos e crustais.

Para arsênio, bismuto, cromo e chumbo, os fluxos de deposição natural representam apenas uma fração das concentrações depositadas na neve. No caso desses elementos, as atividades antrópicas constituem um importante fator responsável por sua mobilização e transporte. $\mathrm{O}$ excesso de concentração de arsênio (59\%), bismuto (10\%), cromo $(39 \%)$ e chumbo $(82 \%)$ provavelmente está relacionado com as atividades antrópicas no Hemisfério Sul.
De acordo com Wolff \& Suttie (1994), as emissões de Pb para a atmosfera do Hemisfério Sul em 1925 foram estimadas em $~ 1.500$ t/ano, com uma grande contribuição proveniente da produção de $\mathrm{Pb}$ na Austrália ( 730 t/ano). Em 1950, as emissões de $\mathrm{Pb}$ chegaram a 4.600 t/ano, com grande contribuição proveniente de aditivos da gasolina ( 1.600 t/ano) e da produção de $\mathrm{Pb}$ na Austrália e no Peru/Brasil ( 1.100 t/ano). Na década de 1980, as emissões de Pb para a atmosfera alcançaram 20.000 t/ano, com as maiores contribuições originadas de aditivos da gasolina ( 12.000 t/ano) e da mineração de chumbo na Austrália, no Brasil e no Peru ( 2.500 t/ano).

Essas informações comparadas com o $\mathrm{FE}_{\mathrm{c}}$ calculado para chumbo mostram que a contribuição das emissões antrópicas e o $\mathrm{FE}_{\mathrm{c}}$ de chumbo aumentaram significativamente entre as décadas de 1970 e 1980, chegando ao auge em 1986. Após esse período, ocorre redução no $\mathrm{FE}_{c}$. Uma das causas da diminuição certamente está ligada à queda no uso de aditivos de $\mathrm{Pb}$ na gasolina a partir de 1980 (Wolff \& Suttie 1994, Barbante et al. 1997).

$\mathrm{O}$ aumento do $\mathrm{FE}_{\mathrm{c}}$ de arsênio entre os anos de 1980 e 1990 se correlaciona com o auge da exploração cuprífera em Chuquicamata $\left(22^{\circ} 19^{\prime} \mathrm{S}, 68^{\circ} 56^{\prime} \mathrm{W}\right)$, no Chile, atingindo o máximo em 1990 e diminuindo posteriormente. A diminuição das taxas de emissão de arsênio durante o processo de fundição não reflete unicamente as condições de intensidade da atividade econômica, mas também é resultado da eficiência e da modernização dos processos de produção. A companhia estatal chilena responsável pelo complexo de Chuquicamata informa que, desde cerca de 1990, implementa políticas de controle e manejo ambientais visando à redução das taxas de emissão atmosféricas do arsênio e de outros poluentes (Codelco 2011).

Emissões antropogênicas de bismuto e cromo são resultado principalmente dos processos de mineração, fundição e refinamento de metais não-ferrosos (Ferrari et al. 2000). 

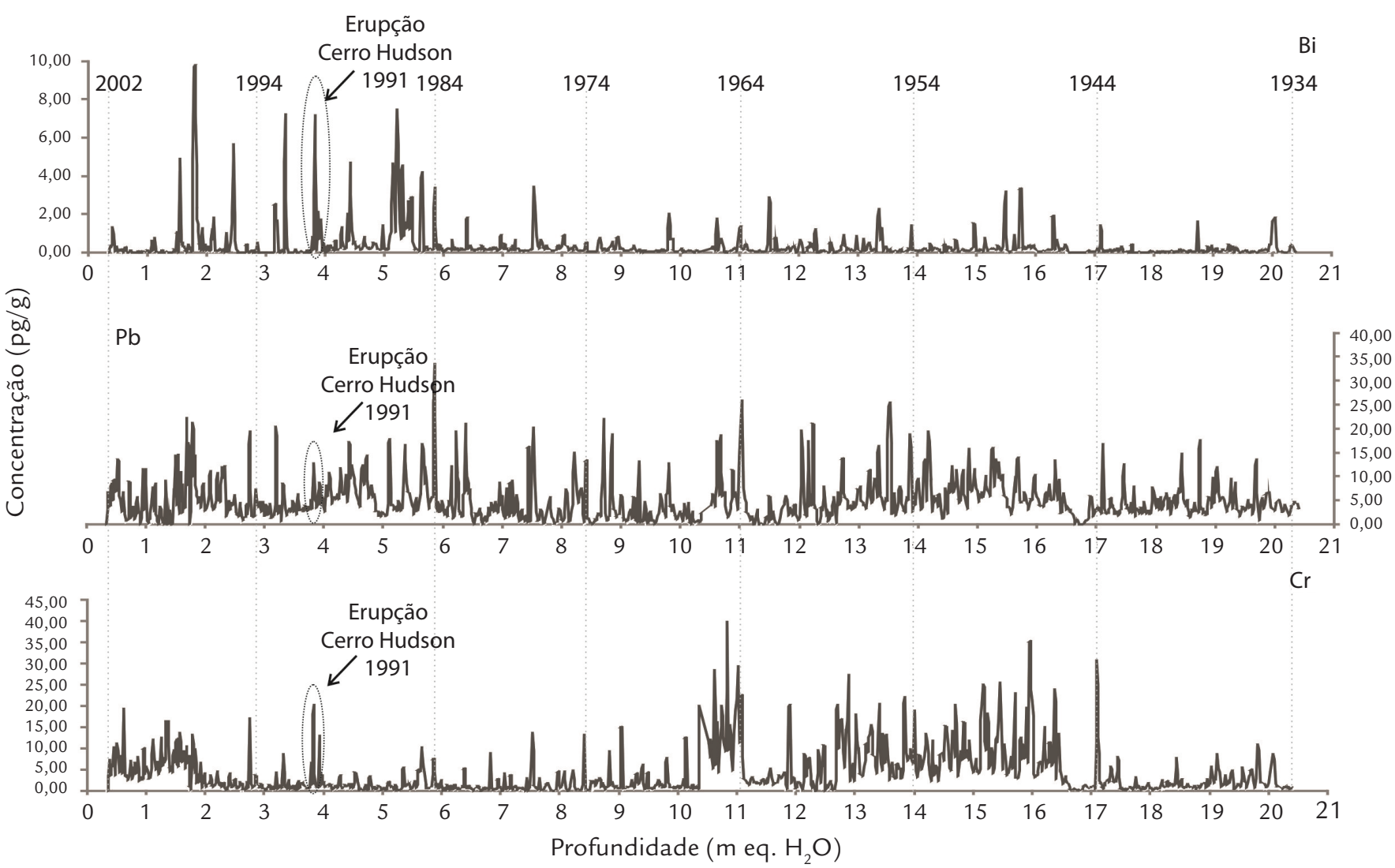

Figura 4

Concentrações de $\mathrm{Bi}, \mathrm{Pb}$ e $\mathrm{Cr}$ medidas no testemunho de firn IC-6. Linhas pontilhadas indicam a idade determinada para o perfil analisado e o círculo pontilhado destaca o pico correspondente à erupção do vulcão Cerro Hudson no Chile.

\section{CONCLUSÕES}

Usando a análise por ICP-MS, foram estudados 16 elementos-traço em amostras de um testemunho de firn da região ocidental da Antártica e utilizados para avaliar as entradas de fontes naturais de emissão de aerossóis, tais como poeira continental, sais marinhos e vulcanismo. Verificou-se que o vulcanismo é uma importante fonte de elementos-traço e é provavelmente a principal fonte de emissão de aerossóis naturais contendo $\mathrm{Bi}, \mathrm{Cd}, \mathrm{Co}$ e $\mathrm{Cr}$. Os teores de concentração de $\mathrm{Al}, \mathrm{Ca}, \mathrm{Fe}, \mathrm{Li}, \mathrm{Mg}, \mathrm{Mn}, \mathrm{Na}, \mathrm{Sr}$ e Ti foram, de maneira geral, classificados como de origem continental ou marinha, ou ainda uma combinação de ambos os insumos. Por outro lado, As e $\mathrm{Pb}$ apresentam, além da contribuição natural continental, um importante incremento associado principalmente a atividades humanas, tais como mineração e transporte no Hemisfério Sul.

\section{AGRADECIMENTOS}

Este trabalho é uma contribuição do Programa Antártico Brasileiro (PROANTAR), financiado com recurso do Conselho Nacional de Desenvolvimento Científico e Tecno-
Algumas das questões abordadas neste trabalho mostram a necessidade de pesquisas mais detalhadas. É necessário um estudo mais aprofundado dos mecanismos que regem os processos de acumulação no testemunho IC-6. Faz-se necessária uma análise detalhada do padrão de circulação das massas de ar atmosféricas na região, bem como dos mecanismos que controlam esse movimento, a fim de se obter um entendimento maior do padrão deposicional encontrado. Por exemplo, pode ser analisado o impacto do fenômeno El Niño - Oscilação Sul (ENOS) sobre o registro elementar obtido. Também são necessários estudos sobre os fluxos de deposição de elementos-traço que participam de ciclos biogeoquímicos naturais. Esses trabalhos são escassos e, no entanto, necessários para a compreensão da contribuição biológica marinha nas concentrações dos elementos analisados.

lógico (CNPq). Jefferson Cardia Simões agradece ao $\mathrm{CNPq}$ pelo apoio financeiro (projeto 557053/2009-9). Franciele Schwanck agradece ao CNPq por sua bolsa de mestrado. 


\section{REFERÊNCIAS}

Barbante C., Bellomi T., Mezzadri G., Cescon P., Scarponi G., Morel C., Jay S., Van de Velde K., Ferrari C., Boutron C.F. 1997. Direct determination of heavy metals at picogram per gram levels in Greenland and Antarctic snow by double focusing inductively coupled plasma mass spectrometry. Journal of Analytical Atomic Spectrometry, 12(9):925-931.

Barbante C., Gabrieli J., Gabrielli P., Vallelonga P., Cozzi G., Turetta C., Hong S., Rosman K., Boutron C.F., Cescon P. 2011. A historical record of heavy metal pollution in alpine snow and ice. In: Quante M., Ebinghaus R., Floser G. (eds.). Persistent Pollution - Past, Present and Future. Springer, Berlin Heidelberg, p. 71-94.

Barbante C., Schwikowski M., Doring T., Gaggeler H.W., Schotterer U., Tobler L., Van De Velde K., Ferrari C., Cozzi G., Turetta A., Rosman K., Bolshov M., Capodaglio G., Cescon P., Boutron C.F. 2004. Historical record of European emissions of heavy metals to the atmosphere since the 1650s from alpine snow/ ice cores drilled near Monte Rosa. Environmental Science \& Technology, 38(15):4085-4090.

Burn-Nunes, L.J., Vallelonga P., Loss R.D., Burton G.R., Moy A., Curran M., Hong S., Smith A.M., Edwards R., Morgan V.I., Rosman K.J.R. 2011. Seasonal variability in the input of lead, barium and indium to Law Dome, Antarctica. Geochimica et Cosmochimica Acta, 75(1):1-20.

Casassa G., Brecher H.H., Cárdenas C., Rivera A. 1998. Mass balance of the Antarctic ice sheet at Patriot Hills. Annals of Glaciology, 27:130-134.

CODELCO - Corporación Nacional del Cobre. Sustentabilidad: Emisiones de Fundiciones, Chile. Disponível em: http://www.codelco.com/emisiones-defundiciones/prontus_codelco/2011-07-11/105820.html. Acessado em nov 2011.

Delmas R.J. 1995. Ice core studies of global biogeochemical cycles. Springer, Berlin Heidelberg, 475 p.

Dixon D.A., Mayewski P.A., Korotkikh E., Sneed S.B., Handley M.J., Introne D.S., Scambos T.A. 2013. Variations in snow and firn chemistry along US ITASE traverses and the effect of surface glazing. The Cryosphere, 7:515-535.

Do Hur S., Cunde X., Hong S., Barbante C., Gabrielli P., Lee K., Boutron C.F., Ming Y. 2007. Seasonal patterns of heavy metal deposition to the snow on Lambert Glacier basin, East Antarctica. Atmospheric Environment, 41(38):8567-8578.

Ferrari C.P., Hong S., Van de Velde K., Boutron C.F., Rudniev S.N., Bolshov M., Chisholm W., Rosman K.J.R.. 2000. Natural and anthropogenic bismuth in Central Greenland. Atmospheric Environment, 34(6):941-948.

Gabrielli P., Barbante C., Boutron C., Cozzi G., Gaspari V., Planchon F., Ferrari C., Turetta C., Hong S., Cescon P. 2005a. Variations in atmospheric trace elements in Dome C (East Antarctica) ice over the last two climatic cycles. Atmospheric Environment, 39(34):6420-6429.

Gabrielli P., Planchon F.A.M., Hong S., Lee K.H., Do Hur S., Barbante C., Ferrari C.P., Petit J.R., Lipenkov V.Y., Cescon P., Boutron C.F. 2005b. Trace elements in Vostok Antarctic ice during the last four climatic cycles. Earth and Planetary Science Letters, 234(1-2):249-259.

Hammes D.F. 2011. Análise e interpretação ambiental da química iônica de um testemunho do manto de gelo da Antártica ocidental. Dissertação de Mestrado, Universidade Federal do Rio Grande do Sul, 106 p.

Hinkley T.K., Lamothe P.J., Wilson S.A., Finnegan D.L., Gerlach T.M. 1999. Metal emissions from Kilauea, and a suggested revision of the estimated worldwide metal output by quiescent degassing of volcanoes. Earth and Planetary Science Letters, 170(3):315-325.

Hong S., Boutron C.F., Edwards R., Morgan V.I. 1998. Heavy metals in Antarctic ice from Law Dome: Initial results. Environmental Research, 78(2):94-103. 
Hong S., Candelone J.P., Patterson C.C., Boutron C.F. 1994. Greenland ice evidence of hemispheric lead pollution two millennia ago by Greek and Roman civilizations. Science, 265(5180):1841-1843.

King J.C. \& Turner J. 1997. Antarctic meteorology and climatology. Cambridge University Press, Cambridge, 425 p.

Knüsel S., Piguet D.E., Schwikowski M., Gaggeler H.W.2003. Accuracy of continuous ice-core trace-element analysis by inductively coupled plasma sector field mass spectrometry. Environmental Science \& Technology, 37(10):2267-2273.

Legrand M. \& Mayewski P. 1997. Glaciochemistry of polar cores: A review. Reviews of Geophysics, 35(3):219-243.

Lide D.R. 2005. Abundance of elements in the Earth's crust and in the sea. In: Lide D. R. (ed). CRC handbook of chemistry and physics, Internet version. CRC Press, Boca Raton.

Marteel A. 2007. Past natural changes in trace elements, rare earth elements (REE), mercury and $\mathrm{Pb}$ isotopes in the EPICA/dome $\mathrm{C}$ ice core (East Antarctica) from 263,000 to 671,000 yrs BP. Tese de Doutorado, Universidade Joseph Fourier (Grenoble1), 279 p.

Martínez-Cortizas A., Pontevedra-Pombal X., García-Rodeja E., Nóvoa-Muñoz J. C., Shotyk W. 1999. Mercury in a spanish peat bog: archive of climate change and atmospheric metal deposition. Science, 284(5416):939-942.

Matsumoto A. \& Hinkley T.K. 2001. Trace metal suites in Antarctic pre-industrial ice are consistent with emissions from quiescent degassing of volcanoes worldwide. Earth and Planetary Science Letters, 186(1):33-43.

Nriagu J.O. 1996. A history of global metal pollution. Science, 272(5259):223-224.

Osterberg E.C. 2007. North Pacific late Holocene climate variability and atmospheric composition. Tese de Doutorado, Universidade do Maine, $161 \mathrm{p}$.

Osterberg E.C., Handley M.J., Sneed S.B., Mayewski P.A., Kreutz K J. 2006. Continuous ice core melter system with discrete sampling for major ion, trace element, and stable isotope analyses. Environmental Science \& Technology, 40(10):3355-3361.

Planchon F.A.M., Boutron C.F., Barbante C., Cozzi G., Gaspari V., Wolff E.W., Ferrari C.P., Cescon P. 2002a. Short-term variations in the occurrence of heavy metals in Antarctic snow from Coats Land since the 1920s. The Science of the Total Environment, 300(1-3):129-142.

Planchon F.A.M., Boutron C.F., Barbante C., Cozzi G., Gaspari V., Wolff E.W., Ferrari C.P., Cescon P. 2002b. Changes in heavy metals in Antarctic snow from Coats Land since the mid-19 $19^{\text {th }}$ to the late- $20^{\text {th }}$ century. Earth and Planetary Science Letters, 200(1-2):207-222.

Renberg I., Brannvall M.L., Bindler R., Emteryd O. 2002. Stable lead isotopes and lake sediments - a useful combination for the study of atmospheric lead pollution history. The Science of the Total Environment, 292(1-2):45-54.

Rhodes R.H. 2011. Insights into Late Holocene climate of the Ross Sea region, Antarctica, from high resolution ice core chemistry. Tese de Doutorado, Victoria University, $231 \mathrm{p}$.

Shotyk W., Weiss D., Appleby P.G., Cheburkin A.K., Frei R., Gloor M., Kramers J.D., Reese S., Van Der Knaap W.O. 1998. History of atmospheric lead deposition since $12,370{ }^{14} \mathrm{C}$ yr BP from a Peat Bog, Jura Mountains, Switzerland. Science, 281(5383):1635-1640.

Simões J.C. 2004. Glossário da língua portuguesa da neve, do gelo e termos correlatos. Pesquisa Antártica Brasileira, 4:119-154.

Simões J. C. 2011. O ambiente antártico: domínio de extremos. In: Simões J.C., Garcia C.A.E., Evangelista H., Campos L.S., Mata M.M., Bremer U.F. (eds.). Antártica e as mudanças globais: um desafio para a humanidade. Edgard Blucher, São Paulo, p. 15-27.

Tao G., Yamada R., Fujikawa Y., Kudo A., Zheng J., Fisher D.A., Koerner R.A. 2001. Determination of trace amounts of heavy metals in Arctic ice core samples using inductively coupled plasma mass spectrometry. Talanta, 55(4):765-772. 
Vallelonga P., Barbante C., Cozzi G., Gaspari V., Candelone J.P., Van de Velde K., Morgan V.I., Rosman K.J.R., Boutron C.F., Cescon P. 2004. Elemental indicators of natural and anthropogenic aerosol inputs to Law Dome, Antarctica.

Annals of Glaciology, 39:169-174.

Vallelonga P., Candelone J.P., Van De Velde K., Curran M.A.J., Morgan V.I., Rosman K.J.R. 2003. Lead, Ba and Bi in Antarctic Law Dome ice corresponding to the $1815 \mathrm{AD}$ Tambora eruption: an assessment of emission sources using

$\mathrm{Pb}$ isotopes. Earth and Planetary Science Letters, 211(3-4):329-341.

Vallelonga P., Van de Velde K., Candelone J.P., Morgan V.I., Boutron C.F., Rosman K.J.R. 2002. The lead pollution history of Law Dome, Antarctica, from isotopic measurements on ice cores: 1500 AD to 1989 AD. Earth and Planetary

Science Letters, 204(1-2):291-306.

Van de Velde K., Vallelonga P., Candelone J.P., Rosman K.J.R., Gaspari V., Cozzi G., Barbante C., Udisti R., Cescon P., Boutron C.F. 2005. Pb isotope record over one century in snow from Victoria Land, Antarctica. Earth and Planetary Science Letters, 232(1-2):95-108.

Wedepohl K. H. 1995. The composition of the continental crust. Geochimica et Cosmochimica Acta, 59(7):1217-1232.

Weller R., Woltjen J., Piel C., Resenberg R, Wagenbach D., König-Langlo G., Kriews M. 2008. Seasonal variability of crustal and marine trace elements in the aerosol at Neumayer station, Antarctica. Tellus, 60B(5):742-752.

Wolff E.W. \& Suttie E.D. 1994. Antarctic snow record of southern hemisphere lead pollution. Geophysical Research Letters, 21(9):781-784.

Wolff E.W., Suttie E.D., Peel D.A. 1999. Antarctic snow record of cadmium, copper, and zinc content during the twentieth century. Atmospheric Environment, 33(10):1535-1541.

Zreda-Gostynska G., Kyle P.R, Finnegan D., Prestbo K.M. 1997. Volcanic gas emissions from Mount Erebus and their impact on the Antarctic environment. Journal of Geophysical Research, 102(B7):15039-15055. 